\title{
3 Research Square

\section{Microglia and infiltrating T-cells adopt long-term, age-specific, transcriptional changes after traumatic brain injury}

Zhangying Chen ( $\sim$ jenniechen2024@u.northwestern.edu )

Northwestern University https://orcid.org/0000-0001-8510-9416

Mecca Islam

Northwestern University

Madeline Timken

Northwestern University

Qinwen Mao

University of Utah School of Medicine

Booker Davis

Northwestern University

Alexios-Fotios Mentis

National and Kapodistrian University of Athens

Steven Schwulst

Northwestern University

Article

Keywords:

Posted Date: January 13th, 2022

DOI: https://doi.org/10.21203/rs.3.rs-1230439/v1

License: (c) (1) This work is licensed under a Creative Commons Attribution 4.0 International License.

Read Full License 


\section{Abstract}

Introduction: Traumatic brain injury (TBI) afflicts over 3 million Americans every year. Patients over 65 years of age suffer increased mortality as well as greater long-term neurocognitive and neuropsychiatric morbidity compared to younger adults. Microglia, the resident macrophages of the brain, are complicit in both. Our published and preliminary data have demonstrated a significant age-effect in which aged microglia are more prone to adopt a constitutively activated state associated with worse neurocognitive and neuropsychiatric outcomes. Therefore, we hypothesized that aged microglia would fail to return to a homeostatic state after TBI but instead adopt a long-term injury-associated state within the brain of aged mice as compared to young-adult mice after TBI. Methods: Young-adult (14-weeks) and aged (80-weeks) C57BL/6 mice underwent TBI via controlled cortical impact vs. sham injury. We utilized single-cell RNA sequencing to examine age-associated cellular responses after TBI. Four months post-TBI or sham injury, brains were harvested, and $\mathrm{CD} 45+$ cells ( $N=4,000$ cells) were isolated via florescence-activated cell sorting. cDNA libraries were prepared via the 10x Genomics Chromium Single Cell 3' Reagent Kit, followed by sequencing on a HiSeq 4000 instrument. The raw data were processed using the Cell Ranger pipeline mapped to the mm10 mouse reference genome and Seurat following standard workflow. Seurat and GOrilla were used for downstream clustering, differential gene expression, and pathway analysis. All cell types were annotated using canonical markers and top expressed genes. ProjecTILs was additionally used to interpret T cell states. Results: Microglia from young-adult and aged mice have distinct transcriptional profiles pre-injury and markedly different transcriptional responses post-injury compared to young-adult mice. Pre-injury, aged mice demonstrated a disproportionate immune cell infiltration, including T cells, as compared to young-adult mice (aged versus young: $45.5 \%$ vs. $14.5 \%$ ). Post-injury, the disparity was amplified with a proportional decrease in homeostatic microglia and greater increased infiltrating T cells compared to young-adult mice (Microglia: $27.5 \%$ vs. $71 \%$; T cell: $45.5 \%$ vs. $4.5 \%$ ). Of note, aged mice post-injury had a subpopulation of unique, age-specific, immune-inflammatory microglia resembling gene profiles of neurodegenerative disease-associated microglia (DAM) with enriched pathways involved in leukocyte recruitment and Alzheimer's disease pathogenesis (FDR < 0.05 ). Contrastingly, post-injury, aged mice demonstrate a heterogenous T-cell infiltration with gene profiles corresponding to CD8 effector memory, CD8 native-like, CD4, and double-negative T cells $(75.9 \%, 2.5 \%$, $12.9 \%$, and $8.6 \%$, respectively) and enriched pathways including tau protein binding, macromolecule synthesis, and cytokine-mediated signaling pathways (FDR < 0.05$)$. Conclusion: We hypothesized that aged microglia would fail to return to a homeostatic state after TBI and adopt a long-term, injuryassociated state within the brain of aged mice as compared to young-adult mice after TBI. In particular, our data suggest an age-dependent reduction of homeostatic microglia post-injury yet an upregulation in a unique microglial subpopulation with a distinct immuno-inflammatory profile. Furthermore, aged subjects demonstrated a markedly disproportionate inflammatory infiltrate after TBI predominated by the presence of CD8+ T cells. In addition, post-injury, brain trauma reorganized the T cell milieu, especially CD8 effector memory T cells, via upregulating genes associated with macromolecule biosynthesis process and negative regulation of neuronal death, possibly linking TBI with its long-term sequelae and complications. Taken together, our data showed that age-specific gene signature changes in the T-cell 
infiltrates and the microglial subpopulation contributes to increased vulnerability of the aged brain to TBI. Age should be an a priori consideration in future TBI clinical trials.

\section{Introduction}

Traumatic brain injury (TBI) is often referred to as a "silent epidemic" (Coburn K, 1992). TBI is an underrecognized global health threat with the highest incidence occurring in North America and Europe (Rusnak, M 2013, Rutland-Brown, 2006). In the United States, the Centers for Disease Control and Prevention estimates that nearly 3 million people sustained a TBI annually, contributing to over one-third of trauma-related deaths, with an estimated annual cost of over $\$ 80$ billion (Corso et al., 2006; Pearson et al., 2012b; Whitlock Jr. and Hamilton, 1995; CDC, 2013; Gardner R et al, 2018). Furthermore, significant long-term complications can occur, resulting in motor, cognitive, and behavioral disorders (Belanger et al., 2016; Carman et al., 2015). The incidence of TBI follows a bimodal distribution primarily afflicting youngadults (15-24 years) and senior citizens (>65 years) (CDC, 2014). In fact, the highest rate of TBI is reported in older patients (defined here-in as $>75$ years) who also have the highest mortality rates, hospitalization rates, and worst functional outcomes after TBI as compared to other age groups (Fraser et al., 2019; Faul, 2010; Rozzenbeek et al., 2013). For long-term survivors of TBI, less than $50 \%$ of older patients become functionally independent as assessed by Glasgow Outcome Scale- Extended (GOS-E) six months after injury (Maidn et al, 2020). Older adults also suffer increased long-term neurocognitive and neuropsychiatric morbidity after TBI as compared to young adults (Maidn et al, 2020).

Recent publications from our laboratory have shown a difference in pathophysiology and behaviors in young adult and aged mice 30 days post-TBI (Islam, et al, 2021). Specifically, at the behavioral level, aged mice have higher level of species-normal anxiety-like behavior and lower exploratory behavior as compared to young adult mice. Additionally, aged mice also have conserved associative learning and memory after TBI as compared to young adult mice. At the pathology level, aged mice have attenuated edema, neuronal loss, and regeneration as well as increased white matter connectivity as compared to young adult mice. In contrast, young adult mice demonstrated signs of neurogenesis after TBI (Islam, et al, 2021). It is evident that aged and young subjects have a differential pathophysiology in response to brain injury. However, the mechanisms of this age-specific difference remain unclear. We surmise that the observed differential pathophysiology between aged and young mice are a result of an aged immune system failing to provide an environment to support repair and regeneration post TBI (Weyand C, 2016).

Microglia, the central nervous system (CNS) resident innate immune cell, participate in a variety of homeostatic CNS functions (Alam A, 2020). They respond to injury immediately and, in doing so, become activated, resulting in a dramatic morphologic transformation, as well as marked changes in gene expression, collectively leading to a cascade of inflammatory events within the brain parenchyma (Loane, 2016). However, some microglia may fail to return to a state of homeostasis and lose certain functions such as motility and phagocytic activity, ultimately resulting in chronic, low-level, neuroinflammation which can contribute to neurodegeneration in the long-term (Loane \& Faden, Izzy S 2019, Block ML, 2007; Gao X, 2008; Streit et al, 2009; Deleidi. 2015). Regardless of injury state, the microglia within aged brains 
can also adopt to a chronic inflammatory state via mechanisms that are only partially understood (Angelova D, et al, 2019). These "primed" microglia are then capable of having an exaggerated inflammatory response to subsequent trauma. Indeed, this exaggerated response to subsequent stimuli may be one of the mechanisms leading to worse outcomes in aged patients after TBI (Keane, L, 2021).

Growing evidence has suggested a gradual T cell infiltration to the CNS with normal aging (Gemechu 2012). In fact, a recent single cell RNA sequencing (scRNA-seq) analysis reveals that a) aged mouse brains have a uniquely enriched $T$ cell infiltration, as compared to their younger counterparts, and $b$ ) these $T$ cells are distinct from $T$ cells within the blood in that they make higher levels of interferon- $\gamma$, a cytokine important for T cell activation (Dulken B, 2019). Whether T cells infiltrate into the aged brain passively via age-related disruptions in the blood brain barrier or actively via antigen recognition has yet to be determined. Furthermore, once T cells infiltrate into the brain parenchyma, little is known about their phenotypes and the signals required to exert downstream events at the site of neuronal damage or inflammation (Schetters et al 2018). Additionally, healthy brains are now known to have clearance pathways such as glymphatic system and meningeal lymphatic system to drain soluble waste proteins and assist immune cells to penetrate the brain parenchyma (Louveau A, et al, 2017). However, with aging, these systems become dysfunctional, potentially leading to the accumulation of T-cell infiltrates in the CNS (Benveniste $\mathrm{H}$, et al, 2019; Mesquita S, et al, 2018). Lastly, whether this age-specific T cell infiltration contributes to the age-related low-grade neuroinflammation remains to be discovered (Deleidi, $M, 2015$ ).

It remains plausible that this T cell infiltration is mediated by age-related signals stemming from microglia, given that the latter are the main antigen-presenting cell (APC) in the brain parenchyma and, in parallel to this, T cell infiltration seems to be associated with brain regions (e.g., white matter) that are linked to age-specific microglia activation (Schetters et al 2018, Moreno-Valladares et al, 2020, Betterman et al, 2021). Indeed, T cells have been identified as a principal cell type generating secondary brain injury in stroke. (Loane 2016, Wnag 2016). Modest T cell infiltration has also been noted in the neuroinflammatory response mediated by microglia in various neurodegenerative diseases, such as Alzheimer disease (AD), Parkinson disease (PD), Amyotrophic Lateral Sclerosis (ALS), and Multiple Sclerosis (MS) (Schetters, 2018, Gonzalez, 2014, Perry 2010, Iba et al 2020). Studies of microglia transcriptomics have also shown an upregulation of genes involved in APC-T cell interactions (Schetters, 2018). In post-mortem TBI patients, T cells appear accumulated within the lesion area one-week post TBI and persist there over time (Dressler, 2007); nonetheless, the exact role of T cells within the aged and injured brain, and whether specific $T$ cell subsets are involved, remains unknown.

Taken together, both normal aging and TBI result in dramatic cellular changes in both microglia and T cells; however, almost all preclinical studies of TBI exclude aged subjects. This has generated a critical unmet need in determining the age-specific mechanisms of differential outcome in aged versus young subjects after TBI. To address this critical unmet need, we utilized our well-established model of TBI in parallel groups of aged and young mice. We aimed to determine the age-specific mechanisms of differential outcome after TBI by isolating microglia and infiltrating immune cells at a chronic time point and then applied single-cell RNA-sequencing approaches to identify unique, age-specific, populations of 
microglia and T cells. We postulated that age-dependent differences in the microglial transcriptome would provide insight into the differential inflammatory infiltrate and functional outcomes observed between age groups. Therefore, we hypothesized that aged microglia would fail to return to a homeostatic state after TBI but instead adopt a long-term injury-associated state within the brain of aged mice as compared to young-adult mice after TBI.

\section{Results}

\section{Histologic Analysis}

We randomly assigned 80-week-old (old) and 14-week-old (young) C57BL/6 male mice (Jackson Laboratories, Bar Harbor, ME, $n=5 /$ group) to either $\mathrm{CCl}$ or to sham injury using established protocols as previously described by our group (Schwulst 2019). Our CCI model of severe TBI generates a significant cavitary lesion in the ipsilateral cortex and hippocampus with surrounding neuronal cell death and morphologic changes in microglia and astrocytes in both ipsilateral and contralateral hemispheres, indicative of microglial activation and astrocyte hypertrophy (Karve et al 2016; Izzy et al, 2019, Fox et al, 1998, Osier et al, 2015). Damage to the hippocampus induces significant cognitive impairment and memory loss (Girgis et al 2016). The hippocampus is comprised of four Cornu Ammonis (CA) subfields and is connected to the dentate gyrus (DG). The CA-DG circuitry plays an important role in synaptic plasticity underlying learning and memory (Arneson et al, 2018). Glial cells are also vital in synaptic plasticity and memory through their interaction with adjacent neurons (Arneson et al, 2018, Todd et al 2006). Histologic analysis of mouse brains from aged and young mice was performed 30 days after either severe TBI or sham injury. Two of the five aged mice died on post-injury day three. None of the young mice died. Brains from the remaining mice were included in the histological analysis. Gross examination and histologic evaluation of animals at 30 days demonstrated similar severity of injury between groups. Significant ipsilateral cortical loss was seen along with both ipsilateral and contralateral deep brain tissue distortion (Figure 1). However, despite identical biomechanical injury parameters, aged mice demonstrated markedly attenuated levels of edema and neurodegeneration as compared to young mice after TBI (Figure 2).

Neurodegeneration was assessed by staining for neuronal nuclei (NeuN), a marker specific for mature and viable neuronal nuclear proteins. Decreased NeuN staining is indicative of a lack of mature neurons. Significant neuronal cell loss was evident in both the cortex and hippocampus at 30 days post TBI (Figure 2A and 2B) in both young and aged mouse brains. However, there was greater neuronal loss in young mouse brains after TBI as compared to aged mouse brains $(p=0.009)$. There was no evidence of neuronal loss in either young or aged mice after sham injury.

CNS injuries evoke a series of cellular responses including the activation of astrocytes and microglia, a process known as "reactive gliosis" (Burda et al 2016). Glial fibrillary acidic protein (GFAP) staining was used to identify gliosis. GFAP stained sections demonstrated severe gliosis in the hippocampus of both young and aged TBI mouse brains. However, when whole brain gliosis was assessed, the brains of young 
TBI mice again demonstrated a greater overall level of gliosis as compared to aged TBI brains $(\mathrm{p}=$ 0.0088). While young TBI mice had markedly more neuronal loss as compared to aged TBI mice, the more extensive gliosis suggests more robust repair and regeneration within the brains of young TBI mice as compared to the brains of aged TBI mice. Microglia were assessed by staining for ionized calciumbinding adaptor molecule 1 (IBA1), a pan-microglia marker that stains both resting and activated cells. As a result, we detected significant IBA1 staining at the injured sites (Figure $3 \mathrm{~A}$ ). In particular, there was significantly more IBA1 staining in the peri-injury brain tissue in aged mice as compared to young TBI mice ( $p=0.004$, Figure 3B). Because expression of IBA1 increases with microglial activation but not the absolute number of either resting or activated microglia (Hopperton et al, 2018), we reasoned that the substantial increase in IBA1 coverage in aged mice post TBI was attributable to increased microglia activation.

Taken together, the histologic data indicates that aged mice have attenuated levels of edema and neurodegeneration, yet prominent reactive gliosis as compared to young mice after identical biomechanical injuries.

\section{scRNA-SeqAnalysis}

The histologic analysis herein reconfirms our previously published data showing that aged mice have attenuated neuronal loss and preserved white matter connectivity after TBI compared to young mice (Loane 2016). In contrast, young mouse brains demonstrate neurogenesis after TBI, while aged mouse brains do not (Loane 2016). As microglia are complicit in all above-mentioned processes (Donat et al, 2017, Chio et al 2015), we reasoned that changes in the microglial transcriptome may reflect unique features of the age associated TBI outcomes. Since microglia also interact with immune cells (e.g., T cells) to promote neuroinflammation, a hallmark of TBI (Schetters 2018, Schimmel et al 2017), we isolated both microglia and infiltrating immune cells from the brains of mice four-months post TBI or sham injury. Since gene regulation determines cellular functions, scrutinizing large-scale genomic changes can reveal molecular determinants that are responsible for such age-specific effects. By isolating microglia and infiltrating immune cells at the single cell level rather than utilizing cell conglomerates, we were able to focus on studying the signals from the most culpable cell types implicated in TBI immunopathogenesis.

To this end, we used 10x Genomics' (Pleasanton, CA) scRNA-seq to determine the transcriptome of microglia and infiltrating immune cells isolated from aged male mice $(n=4$, two pooled mice per group, 80-weeks) at four months post-TBI or sham injury. These data were then compared to those from cells derived from brains that were, in turn, isolated from young-adult male mice $(n=4$, two pooled mice per group, 14-weeks) at four months post-TBI or sham injury (Figure 4A). In total, 14,247 cells from 8 mice were sequenced: 4,216 cells from young mice undergoing sham surgery, 2,532 cells from young mice undergoing $\mathrm{TBI}, 4,080$ cells from aged mice undergoing sham surgery, and 3,419 cells from aged mice undergoing TBI. After quality control and adjusting for noise, a range of $10 \% \sim 30 \%$ of cells were removed (Table 1). 


\begin{tabular}{|llllllll|}
\hline Age & $\begin{array}{l}\text { TBI } \\
\text { status }\end{array}$ & $\begin{array}{l}\text { Cells } \\
\text { sequenced }\end{array}$ & $\begin{array}{l}\text { Median } \\
\text { genes per } \\
\text { cells }\end{array}$ & $\begin{array}{l}\text { Mean } \\
\text { reads } \\
\text { per cell }\end{array}$ & $\begin{array}{l}\text { Total no. of } \\
\text { cells } \\
\text { (after QC) }\end{array}$ & $\begin{array}{l}\text { Total no. } \\
\text { of } \\
\text { microglia }\end{array}$ & $\begin{array}{l}\text { Total no. of } \\
\text { cells }\end{array}$ \\
\hline Young & Sham & 4,216 & 720 & 74,483 & 2847 & 1551 & 195 \\
\hline Young & TBI & 2,532 & 1,713 & 118,784 & 1651 & 1084 & 70 \\
\hline Aged & Sham & 4,080 & 1,270 & 72,436 & 3747 & 1893 & 1000 \\
\hline Aged & TBI & 3,419 & 1,520 & 88,262 & 2891 & 777 & 1274 \\
\hline
\end{tabular}

Table 1. Sample information.

By visualizing the data in two dimensions through Uniform Manifold Approximation and Projection (UMAP), we observed the transcriptomic distribution of computed clusters (Figure 4B). To characterize the cell identity of the computed clusters, we utilized the immune cell markers (fig. S1) curated from the literature (Allen Brain Atlas, UCSC Cell Browser, PanglaoDB, Hammondet al, Masuda et al, and Ochocka et al) to annotate individual cell identity. The majority of cells were identified as microglia (MG) and T cells, and the minor populations include CNS border-associated macrophages (CAM), natural killer (NK) cells, monocytes or monocyte-derived macrophages (MoMФ), neutrophils (NP), and unknown cells specific to young subjects (Figure 4B). These young-specific unknown cells (Y.UK) are characterized by a high expression of genes associated with Scd2, a marker for pre-microglia (Szulzewsky, et al, 2016; Ochocka et al, 2021), and genes encoding proliferation-related proteins (such as Prkcz, Tubb3 and Rit2) (Shi, et al 2013). More significantly, we identified a striking disparity between aged mice and young mice in their immune response post brain injury. In particular, pre-injury, aged mouse brains demonstrated an increased proportion of infiltrating immune cells including T-cells, B-cells, and NK-cells as compared to the brains of young-adult mice (T-cells: $28 \%$ vs $7.5 \%$; B-cells: $6 \%$ vs $1.5 \%$; NK-cells: $3.5 \%$ vs $0.5 \%$ ) (Figure 4C). Post-injury, aged mice had a markedly different composition of infiltrating immune cells within the injured brain compared to young-adult mice at 4 months post-TBI. Most notable was a significantly higher proportion of T-cell infiltrates into the injured brain parenchyma of aged mice compared to the brains of young-adult mice after TBI (45.5\% vs 4.5\%) (Figure 4C). Additionally, the percentage of microglia demonstrated a differential response to injury between young and aged mice. Post-injury, young mice increased their proportion of microglia, which might be due to decreased proportion of Y.UK (MG: $71 \%$ vs $58 \%$; Y.UK: $14 \%$ vs $28.5 \%$ ). Given that Y.UK had an upregulation of genes encoding premicroglia, this young-specific cluster may reflect maintenance properties of microglia. In contrast, aged mice had a proportionately greater decrease in microglia post-injury, although their proportion decreased due to infiltration of T-cells (MG: $27.5 \%$ vs $53 \%$ ). Taken together, these results demonstrate dynamic changes in immune cell composition in young versus aged mouse brains both at baseline as well as after injury.

\section{Assessment of T-cell subset}

To further identify the molecular features contributing to the age-specific differential response post-TBI, we performed scRNA-seq analyses on major cell subpopulations, namely T-cells and MG. Our analysis 
showed that aged sham mice have a marked recruitment of infiltrating immune cells, composed of predominantly T-cell infiltrates, compared to young sham mice (Figure 4C). By further annotating different T subtypes using canonical markers and ProjecTILS, a R package combining reference single-cell atlases to interpretation of $T$ cell states (Andreattae, et al, 2021; Figure 5A; fig. S2), we noted that $T$ cells in aged sham groups are mainly composed of $\mathrm{CD}^{+} \mathrm{T}$ cells, which include both effector memory (EM) and naïvelike (i.e., century memory and naïve; NL) $C D 8^{+} T$ cells, followed by CD4 and double negative $T$ (DN) T cells (number of cells annotated: CD8 ${ }^{+}$EM: $n=1784, C D 8$ NL: $n=79, C D 4: n=248, D N T: n=154 ;$ Fig. 5A and Fig. 5B), as previously reported (Ritzel R, et al, 2016, Dulken B, 2019, Panwar, A et al, 2020, Moreno-Valladares, $M$ et al, 2020). Notably, we identified that, four months post-injury, this T-cell infiltration expands with an even greater percentage of CD8, CD4, and DN T-cells (post- vs pre-injury in aged mice: CD8 EM: $51.8 \%$ vs $46.4 \%$; CD8 NL: $39.2 \%$ vs $38.0 \%$; CD4: $63.3 \%$ vs $29.8 \%$; DNT: $68.2 \%$ vs $25.3 \%$; Fig. 5 C). This is in striking comparison to young adult mice, where only a small percentage of the inflammatory infiltrate comprising T-cells regardless of injury status (post- vs pre-injury in aged mice: CD8 EM: $51.8 \%$ vs $46.4 \%$; CD8 NL: $39.2 \%$ vs $38.0 \%$; CD 4 : $63.3 \%$ vs $29.8 \%$; DNT: $68.2 \%$ vs $25.3 \%$; Fig. 5 C). Comparing RNA transcriptomes of CD8 EM T cells from aged sham and aged TBI by differential expression showed increased expression of cytokine and jun kinase (JNK) kinase signaling transduction molecules Jak1 (encoding janus kinase 1) and Fos (encoding fox proto-oncogene) in aged TBI mice as compared to aged sham mice (Kaech $S$, et al, 2002; Fig. 5D). Interestingly, CD8 EM T cells from aged TBI mice had decreased expression of genes associated with essential $\mathrm{T}$ cell functions such as the $\mathrm{T}$ cell activation $C D 69$, iron regulation Fth (encoding ferritin heavy chain), calcium binding S100a6 (encoding S100 calcium binding protein A6), and adhesion molecules Vim (encoding Vimentin) (Cronin S, et al, 2019, Szabo P, et al, 2019) compared to aged sham mice. These findings suggest that CD8 EM T cells from aged mice adopted a functional change long-term post-injury. As for CD8 NL T cells from aged TBI mice, they preferentially upregulated genes involving in cellular migration such as Dock2 (encoding dedicator of cytokinesis 2), Macf1 (encoding actin cross-linking factor 1 ), T cell growth and proliferation TSN (encoding tumor supernatant) and SRSF5 (encoding serine and arginine rich splicing factor 5), and downregulated genes involved in apoptosis and quiescence such as $B c / 2$ (encoding $B$ cell lymphoma) and Klf2 (encoding kruppel-like factor 2) as compared to aged sham mice (Nishihara, $\mathrm{H}$ et al, 2002, Chen, $\mathrm{H}$ et al, 2006, Sebzda, E et al, 2008; Fig. 5E). Differential expression analysis of CD4 and DN T showed upregulation of Egr1 (encoding early growth response 1), a stimulator for T-cell activation and IL-2 production and genes encoding surface chains such as $C d 3 e, C d 3 g$ (Bird, L, 2007; fig. S3). Analysis of pathways on differentially expressed genes in CD8 EM T cells from aged TBI mice contained enriched pathways including tau protein binding, macromolecule biosynthesis process, and negative regulation of neuronal death (Fig. 5F), which supported previous findings that aged mice have attenuated neuronal loss as compared to young adult mice and CD8 EM T cells might play a pathological role post-injury (Islam M, et al, 2021). Analysis of pathways on differentially expressed genes in CD8 NL T cells contained enriched pathways, including cytokine-mediated signaling pathway and positive regulation of mRNA splicing (Fig. 5G). Thus, enhanced T cell proliferation and cytokine signaling can be observed in CD8 NL T cells from aged TBI mice. 
A moderate T-cell infiltration, particularly CD8 T cells, is a known age-related phenomenon, damaging the brain by potentially modulating synaptic plasticity, potentiating inflammation, and contributing to cognitive decline (Ritzel R, et al, 2016, Unger MS, et al, 2020, Betterman K et al, 2021). Our data indicates that brain trauma further triggers the accumulation of T cell-cell infiltrates, which lasted up to four-month post-injury. Additionally, brain trauma reorganized the T cell milieu, especially CD8 EM T cells, via upregulating genes associated with macromolecule biosynthesis process and negative regulation of neuronal death, possibly linking TBI with its long-term sequelae and complications. Taken together, these observations potentially explain the differential response we previously observed between young and aged subjects post-TBI and could be of clinical relevance (Islam M, et al, 2021).

\section{Assessment of Microglia (MG) Subset}

In the subsequent scRNA-seq analysis of the microglia population, we identified subclusters of microglia that originated predominantly from either young adult mice or aged mice (Fig. 6A). Based on these observations, five subsets of microglial cells with distinct transcriptional profiles were identified: young mice-enriched microglial subcluster 1 (Young MG1), young mice-enriched microglial subcluster 2 (Young MG2), aged mice-enriched microglial subcluster 1 (Aged MG1), aged mice-enriched microglial subcluster 2 (Aged MG2), and microglial subclusters with no dominant origins (MG1). Differential expression analysis demonstrated that Aged MG1 adopts gene signature of DAM, a novel microglia type associated with neurodegenerative diseases (Keren-Shaul, $\mathrm{H}$, et al, 2017). Specifically, this subcluster presented a notable gene signature with high expression level Apoe (encoding apolipoprotein E), Cst7 (encoding cystatin-F), Lp/ (encoding lipoprotein lipase) (Keren-Shaul, $\mathrm{H}$, et al, 2017) shared by both aging microglia and DAM (Knag SS, et al, 2018, Keane L, et al, 2021). Additionally, Aged MG1 had high expression of Lgals3 (encoding galectin), Lyz2 (encoding lysozyme 2), Cd63 (encoding an exosome marker CD63 antigen), as well as MIF-CD74 signaling molecules Mif (encoding macrophage migration inhibitory factor), and $C D 74$ (encoding $\mathrm{H}-2$ class II histocompatibility antigen y chain), indicating that this Aged MG2 is associated with immune-inflammatory responses in aged brains (Ghoochani, A, et al, 2016, Jin C, et al, 2021). Likewise, MG1 and young MG1 subclusters had high expression of genes associated with microglial cell activation and chemokine-related inflammation such as PIp1 (encoding proteolipid protein 1), $C c / 3$ (encoding $\mathrm{C}-\mathrm{C}$ motif chemokine ligand 3), $\mathrm{Ccl} 4$ (encoding $\mathrm{C}-\mathrm{C}$ motif chemokine ligand 4), and Cc/12 (encoding C-C motif chemokine ligand 12) (Hammond et al, 2019, Tatar C, et al, 2010). On the contrary, differential expression analysis demonstrated that Aged MG2 subcluster had high expression of microglial genes, especially homeostatic markers, including P2ry 12 (encoding purinergic receptor P2Y12), CX3cr1 (encoding C-X3-C motif chemokine receptor 1), Lgmn (encoding legumain), and Tmem 119 (encoding transmembrane protein 110). In contrast, young MG2 subcluster had high expression of immediate early genes such as Jun (encoding transcription factor AP-1), Junb (encoding transcription factor jun-B), Egr1 (Ochocka, N, 2021). Post-injury, we observed a reduction in the proportion of microglia from aged mice in most microglial subclusters, but this might be due to increased T-cell infiltration (Fig. 6B). 
To further elucidate the roles of microglia in the differential post-TBI outcomes seen in aged vs young subjects, we examined expression levels of highly upregulated genes in microglia from aged mice post injury (significantly upregulated genes in aged TBI compared to aged sham) versus young mice post injury (significantly upregulated genes in young TBI as compared to young sham). Common $(n=212)$ and sample-specific ( $n=111$ in Aged_TBI and $n=1098$ in Young_TBI) highly upregulated genes were identified and compared. Consequently, we found that the majority of genes upregulated in the aged TBI brains are also expressed by young TBI brains and their RNA expression levels are even higher in young TBI brains than old TBI brains (Fig. 6C). Among them, we identified CCr5, a gene encoding a chemokine receptor mainly distributed in microglia and known to recruit T cells during inflammation under disease status (Joy et al., 2019, Huerta et al., 2004, Li T, et al, 2019). This finding correlates to the noted increased percentage of T-cells in the brains of aged mice post-injury. We also identified $L r p 1$, encoding a major receptor for APOE and amyloid- $\beta(A \beta)$, linking TBI with AD pathogenesis (Yang et al, 2016). Microglia from Aged_TBI also particularly upregulated genes such as Rock1 (encoding a serine/threonine kinase), Pdcd10 (encoding programmed cell death protein 10), I/1a (encoding interleukin-1), Ifngr1 (encoding interferon-gamma receptor 1 ), contributing to post TBI microglia-mediated neuroinflammation (Mulherkar et al, 2020, Todd B et al, 2021). Downstream pathway analysis of these highly upregulated genes in aged TBI contained enriched pathways including leukocyte migration involved in inflammatory response, amyloid-beta clearance, positive regulation of nervous system development, and negative regulation of cell death (Fig. 6D), which supported our previous findings that aged mice have attenuated neuronal loss as compared to young adult mice (Islam M, et al, 2021) and indicated that, through this updated study, aged microglia adopted a functional change long-term post TBI potentially contributing to the agespecific TBI outcomes.

\section{Conclusion}

We hypothesized that aged microglia would fail to return to a homeostatic state after TBI but instead adopt a long-term, injury-associated state within the brain of aged mice as compared to young-adult mice after TBI. Our findings indicate that the aged brain exhibits greater vulnerability to brain trauma due to aged immune system, resulting in chronically upregulated immune cell infiltrates and non-homeostatic, injury-associated inflammatory microglia. Notedly, pre-injury, aged mice demonstrated an increased proportion of infiltrating immune cells, particularly T cells compared to young-adult mice. Post-injury, this disparity was amplified with a greater proportionally increased T-cell infiltrates. These proportionally increased T-cell infiltrates had gene profiles corresponding to CD8 EM, CD4, and DN T cells with enriched pathways including tau protein binding, macromolecule synthesis, and cytokine-mediated signaling pathways. Post-injury, the proportions of microglia became markedly decreased in aged brains compared to young brains. Nevertheless, we identified a subpopulation of age-specific, inflammatory microglia which phenocopied that of DAM by upregulating a variety of genes such as Apoe4, Cst7, and Lpl. Additionally, this microglia subpopulation established a distinct immuno-inflammatory profile with top markers including Lgals3, Mif, Cd74, Lyz2, as well as known age-related ribosome and exosome markers. 
Post-injury, this microglia subpopulation adopted an injury-dependent state upregulating genes with functional implications in leukocyte recruitment, AD pathogenesis, and neuroinflammation.

As a crucial effector element of the adaptive immune system in the CNS, a group of infiltrated T cells have been previously noted as pathogenic and induce inflammation in experimental autoimmune encephalomyelitis (EAE) mice that model MS (Fletcher JM). Yet, studies have also indicated that some infiltrated $T$ cells can limit neuronal damage caused by mechanical injury and neurodegeneration (Ellwardt E, 2016). Depending on the brain condition and infiltrated T cell subtypes and lineage, $T$ cells can play both destructive and beneficial roles (Schetters, 2018); however, what regulates this balance remains unknown. Our study indicates that aging can impact this balance by marked recruitment of CD $8^{+}$ cytotoxic $\mathrm{T}$ cells. Recent reports have described that $\mathrm{CD} 8^{+} \mathrm{T}$ cells cause a long-term neurological impairment after TBI, which align with our findings in single cell- transcriptomics, and they support our published data demonstrating worse behavioral outcomes in aged mice post-TBI as compared to their young counterparts (Daglas, 2019, Islam M, 2021). The findings of increased CD4 and DN T infiltrates in the mouse brains are also equally intriguing, as their roles in aging have been recently uncovered. Specifically, CD4 T-cells become more regulatory and cytotoxic, contributing to chronic inflammation and immunity decline with age (Elyahu Y, 2019). Likewise, increased DN T-cell infiltrates, though comprising only 1 to $5 \%$ of the total T cell population, have been associated with neuroinflammation and might cause further brain damage (Ford S, 2007, Young KJ, 2003, Brandt D 2018, Meng H, 2019). Overall, our data depict a complex and dynamic landscape of age-specific T-cell infiltrates, which might be the associated with differential injury outcomes between aged and young subjects.

In healthy brains, microglia protect neurons and react to counteract any disturbances in immunological homeostasis (Szalay G, et al, 2016). With age, microglia are primed to react abnormally and become neurotoxic and destructive (Luo, X, et al, 2010). In line with several recent scRNA-seq studies, our study also demonstrated a unique, age-specific, immune-inflammatory microglia subset resembling gene profiles of DAM which might be responsible for microglial senescence and dysfunctions in aged subjects (Hammond 2019, Jin C 2021, Sala-Frigerio, 2019). Importantly, our results indicated that this microglia subset might be the culprit for differential TBI outcomes seen in aged subjects as compared to their younger counterparts. Long-term post-TBI, this subset upregulates genes enriched in pathways such as leukocyte migration involved in the inflammatory response, amyloid-beta clearance, positive regulation of nervous system development, and negative regulation of cell death. Since microglia are known for their transcriptomic heterogeneity on a temporal and spatial axis, our findings illuminate an important aspect of microglial diversity in connection with aging and help identify a specific subset with specialized functions in the context of TBI (Masuda, T, et al, 2020). Nevertheless, whether this microglia subset is the dominant driver for the age-effect post-TBI remains to be elucidated. Additionally, how these age-specific microglia become activated in the aged brain remains understudied. So far, one study reported that the cell surface receptor, CD74, may function as a specific signal transducer in microglia of aged brains (Jin C 2021, Sala-Frigerio, 2019). CD74 is the central receptor for MIF, which has been found to be significantly upregulated in the brains of aged subjects, and its level is a potential biomarker for early-stage $A D$, 
contributing to glial activation, cognitive impairment, and tau pathology (Nasiri E, 2020). Thus, we reason that TBI may further induce enhanced CD74-MIF in addition to recruiting peripheral immune cells to the brain to evoke worse neuropsychiatric and neurocognitive outcomes seen in aged subjects.

Several limitations of the present study should be noted. For instance, since microglia are known for their heterogeneity on a spatial axis, our study failed to capture the proper spatial representation of different microglia subsets. To overcome this hurdle, future studies can integrate spatial information with scRNAseq to extract more location-dependent transcriptional profiles for investigating downstream events (Lahnemann et al, 2020). Nevertheless, we argue that understanding the molecular differences at the single-cell level in aged versus young mice affected by TBI has the potential to provide one of the rationales for clinical observations that, i.e., geriatric patients have worse TBI outcomes than younger patients. Compared to the conventional bulk RNA analyses, scRNA-seq is capable of studying microglia multiplicity comprehensively in an unbiased fashion and identifying disease- or injury-directed subsets (Masuda, 2020). Quantifying microglia heterogeneity has the potential to predict TBI outcomes as microglia in TBI result in different phenotypes, manifested as different morphologies and gene expression, depending on chronicity and the stage of the injury (Donat C, et al, 2017). Additionally, uncovering microglia heterogeneity will have a broad implication for neurodegenerative diseases as both microglia activation and microglia senescence have been reported as mechanisms for neurodegeneration (Hickman S, 2018, Angelova D, 2019). Another pitfall of our current study is that we failed to detect different $T$ cell lineages (i.e., $\gamma \delta$ versus $\alpha \beta T$ ). To the best of our knowledge, detecting $\gamma \delta T$ cells from $\alpha \beta T$ cells is limited utilizing scRNA-seq, in both human and animal studies, as detection of $\gamma \delta T$ cells requires not only large sample sizes but also a signature of $\gamma \delta \mathrm{T}$ cells combined with NK and T CD $8^{+}$-discarding genes (Pizzolato, 2019). To overcome this, we can detect T cell lineages via flow cytometric assessment or single-cell T-cell Receptor (TCR) sequencing in future studies (Lee, M, et al, 2020, Gherardin, N, et al, 2021). Understanding $T$ cell lineages will reveal crucial insight into the pathology of TBI, as $\gamma \delta T$ cells have been known to promote the inflammatory responses in the CNS (Wo, J, et al, 2020). Lastly, we excluded female mouse brains in the present study as sex, in general, and estrous cycle, in particular, results in differential TBI outcomes. Future studies should include female mice, which will add additional value to the field, as there are currently no available scRNA-seq datasets from geriatric female mouse brains (Jin et al 2021). Overall, our study unmasks cellular composition changes and transcriptome alterations in response to TBI in aged versus young mice. This has been an understudied field in current research efforts, as age is the primary confounder in the context of injury or chronic disease and adds complexity to the investigation of biology. However, the study of age-effect in TBI is of the utmost importance as older patients suffer the highest mortality and greatest long-term neurocognitive and neuropsychiatric morbidity compared among all the age groups (Fraser et al., 2019; Faul, 2010; Rozzenbeek et al., 2013).

Taken together, our data explain the differential response between young and aged subjects post-TBI and could be of medically relevant extrapolations. Future studies will focus on further quantifying identified $T$ subtypes and elucidating their functional roles in this age-effect. Furthermore, as T-cells infiltrate in

Page 12/27 
connection to heightened microglial reactivity and exacerbated cognitive decline, it is crucial to further uncover the interactions between microglia and infiltrating T-cells or their subtypes, which could ultimately lead to a potential age-specific therapeutic development (Betterman K, 2021).

\section{References}

Alam, A., Thelin, E. P., Tajsic, T., Khan, D. Z., Khellaf, A., Patani, R., \& Helmy, A. (2020). Cellular infiltration in traumatic brain injury. J Neuroinflammation, 17(1), 328. doi:10.1186/s12974-020-02005-x

Andreatta, M., Corria-Osorio, J., Muller, S., Cubas, R., Coukos, G., \& Carmona, S. J. (2021). Interpretation of T cell states from single-cell transcriptomics data using reference atlases. Nat Commun, 12(1), 2965. doi:10.1038/s41467-021-23324-4

Angelova, D. M., \& Brown, D. R. (2019). Microglia and the aging brain: are senescent microglia the key to neurodegeneration? J Neurochem, 151(6), 676-688. doi:10.1111/jnc.14860

Arneson, D., Zhang, G., Ying, Z., Zhuang, Y., Byun, H. R., Ahn, I. S., . . Yang, X. (2018). Single cell molecular alterations reveal target cells and pathways of concussive brain injury. Nat Commun, 9(1), 3894. doi:10.1038/s41467-018-06222-0

Batterman, K. V., Cabrera, P. E., Moore, T. L., \& Rosene, D. L. (2021). T Cells Actively Infiltrate the White Matter of the Aging Monkey Brain in Relation to Increased Microglial Reactivity and Cognitive Decline. Front Immunol, 12, 607691. doi:10.3389/fimmu.2021.607691

Belanger, H. G., Vanderploeg, R. D., \& McAllister, T. (2016). Subconcussive Blows to the Head: A Formative Review of Short-term Clinical Outcomes. J Head Trauma Rehabil, 31(3), 159-166. doi:10.1097/HTR.0000000000000138

Benveniste, H., Liu, X., Koundal, S., Sanggaard, S., Lee, H., \& Wardlaw, J. (2019). The Glymphatic System and Waste Clearance with Brain Aging: A Review. Gerontology, 65(2), 106-119. doi:10.1159/000490349

Bird, L. A stimulating collaboration. Nat Rev Immuno/ 7, 4 (2007). https://doi.org/10.1038/nri2008

Blighe, K, S Rana, and M Lewis. 2018. "EnhancedVolcano: Publication-ready volcano plots with enhanced colouring and labeling." https://github.com/kevinblighe/EnhancedVolcano.

Block, M. L., \& Hong, J. S. (2007). Chronic microglial activation and progressive dopaminergic neurotoxicity. Biochem Soc Trans, 35(Pt 5), 1127-1132. doi:10.1042/BST0351127

Brandt, D., \& Hedrich, C. M. (2018). TCRalphabeta(+)CD3(+)CD4(-)CD8(-) (double negative) T cells in autoimmunity. Autoimmun Rev, 17(4), 422-430. doi:10.1016/j.autrev.2018.02.001

Burda, J. E., Bernstein, A. M., \& Sofroniew, M. V. (2016). Astrocyte roles in traumatic brain injury. Exp Neurol, 275 Pt 3, 305-315. doi:10.1016/j.expneurol.2015.03.020 
Carman, A. J., Ferguson, R., Cantu, R., Comstock, R. D., Dacks, P. A., DeKosky, S. T., . . Fillit, H. M. (2015). Expert consensus document: Mind the gaps-advancing research into short-term and long-term neuropsychological outcomes of youth sports-related concussions. Nat Rev Neurol, 11(4), 230-244. doi:10.1038/nrneurol.2015.30

Centers for Disease Control and Prevention (CDC), Traumatic Brain Injury (TBI): Incidence and Distribution, 2014.Introduction to Brain Injury - Facts and Stats, February 2000

Centers for Disease Control and Prevention (CDC). (2013). CDC grand rounds: reducing severe traumatic brain injury in the United States. MMWR Morb. Mortal. Wkly. Rep. 62, 549-552

Chen, H. J., Lin, C. M., Lin, C. S., Perez-Olle, R., Leung, C. L., \& Liem, R. K. (2006). The role of microtubule actin cross-linking factor 1 (MACF1) in the Wnt signaling pathway. Genes Dev, 20(14), 1933-1945. doi:10.1101/gad.1411206

Chenghao Jin , Yijie Shao, Xiaotao Zhang , Jiani Xiang , Ruize Zhang , Zeyu Sun , Shuhao Mei , Jingyi Zhou , Jianmin Zhang, Ligen Shi. A Unique Type of Highly-Activated Microglia Evoking Brain Inflammation via Mif/Cd74 Signaling Axis in Aged Mice. Aging and disease. 2021, 12(8): 2125-2139 https://doi.org/10.14336/AD.2021.0520

Chio, C. C., Lin, M. T., \& Chang, C. P. (2015). Microglial activation as a compelling target for treating acute traumatic brain injury. Curr Med Chem, 22(6), 759-770. doi:10.2174/0929867321666141106124657

Coburn, K. (1992). Traumatic brain injury: the silent epidemic. AACN Clin Issues Crit Care Nurs, 3(1), 9-18. doi:10.4037/15597768-1992-1002

Corso, P., Finkelstein, E., Miller, T., Fiebelkorn, I., \& Zaloshnja, E. (2006). Incidence and lifetime costs of injuries in the United States. Inj Prev, 12(4), 212-218. doi:10.1136/ip.2005.010983

Cronin, S. J. F., Woolf, C. J., Weiss, G., \& Penninger, J. M. (2019). The Role of Iron Regulation in Immunometabolism and Immune-Related Disease. Front Mol Biosci, 6, 116.

doi:10.3389/fmolb.2019.00116

Da Mesquita, S., Louveau, A., Vaccari, A., Smirnov, I., Cornelison, R. C., Kingsmore, K. M., . . Kipnis, J. (2018). Functional aspects of meningeal lymphatics in ageing and Alzheimer's disease. Nature, 560(7717), 185-191. doi:10.1038/s41586-018-0368-8

Daglas, M., Draxler, D. F., Ho, H., McCutcheon, F., Galle, A., Au, A. E., . . Medcalf, R. L. (2019). Activated CD8(+) T Cells Cause Long-Term Neurological Impairment after Traumatic Brain Injury in Mice. Cell Rep, 29(5), 1178-1191 e1176. doi:10.1016/j.celrep.2019.09.046

Deleidi, M., Jaggle, M., \& Rubino, G. (2015). Immune aging, dysmetabolism, and inflammation in neurological diseases. Front Neurosci, 9, 172. doi:10.3389/fnins.2015.00172 
Donat, C. K., Scott, G., Gentleman, S. M., \& Sastre, M. (2017). Microglial Activation in Traumatic Brain Injury. Front Aging Neurosci, 9, 208. doi:10.3389/fnagi.2017.00208

Eden, E., Lipson, D., Yogev, S., \& Yakhini, Z. (2007). Discovering motifs in ranked lists of DNA sequences. PLoS Comput Biol, 3(3), e39. doi:10.1371/journal.pcbi.0030039

Eden, E., Navon, R., Steinfeld, I., Lipson, D., \& Yakhini, Z. (2009). GOrilla: a tool for discovery and visualization of enriched GO terms in ranked gene lists. BMC Bioinformatics, 10, 48. doi:10.1186/14712105-10-48

Ellwardt, E., Walsh, J. T., Kipnis, J., \& Zipp, F. (2016). Understanding the Role of T Cells in CNS Homeostasis. Trends Immunol, 37(2), 154-165. doi:10.1016/j.it.2015.12.008

Elyahu, Y., Hekselman, I., Eizenberg-Magar, I., Berner, O., Strominger, I., Schiller, M., . . Monsonego, A. (2019). Aging promotes reorganization of the CD4 T cell landscape toward extreme regulatory and effector phenotypes. Sci Adv, 5(8), eaaw8330. doi:10.1126/sciadv.aaw8330

Faul, Mark;Wald, Marlena M.;Xu, Likang;Coronado, Victor G.;Corporate Authors(s) : National Center for Injury Prevention and Control (U.S.), Division of Injury Response. Traumatic brain injury in the United States; emergency department visits, hospitalizations, and deaths, 2002-2006. 2010. URL : https://stacks.cdc.gov/view/cdc/5571

Finak, G., McDavid, A., Yajima, M., Deng, J., Gersuk, V., Shalek, A. K., .. Gottardo, R. (2015). MAST: a flexible statistical framework for assessing transcriptional changes and characterizing heterogeneity in single-cell RNA sequencing data. Genome Biol, 16, 278. doi:10.1186/s13059-015-0844-5

Ford, M. S., Chen, W., Wong, S., Li, C., Vanama, R., Elford, A. R., .. . Zhang, L. (2007). Peptide-activated double-negative $T$ cells can prevent autoimmune type-1 diabetes development. Eur J Immunol, 37(8), 2234-2241. doi:10.1002/eji.200636991

Fox, G. B., Fan, L., Levasseur, R. A., \& Faden, A. I. (1998). Sustained sensory/motor and cognitive deficits with neuronal apoptosis following controlled cortical impact brain injury in the mouse. J Neurotrauma, 15(8), 599-614. doi:10.1089/neu.1998.15.599

Franzen, O., Gan, L. M., \& Bjorkegren, J. L. M. (2019). PanglaoDB: a web server for exploration of mouse and human single-cell RNA sequencing data. Database (Oxford), 2019. doi:10.1093/database/baz046

Fraser, E. E., Downing, M. G., Biernacki, K., McKenzie, D. P., \& Ponsford, J. L. (2019). Cognitive Reserve and Age Predict Cognitive Recovery after Mild to Severe Traumatic Brain Injury. J Neurotrauma, 36(19), 27532761. doi:10.1089/neu.2019.6430

Gao, X., Zhang, H., Takahashi, T., Hsieh, J., Liao, J., Steinberg, G. K., \& Zhao, H. (2008). The Akt signaling pathway contributes to postconditioning's protection against stroke; the protection is associated with the MAPK and PKC pathways. J Neurochem, 105(3), 943-955. doi:10.1111/j.1471-4159.2008.05218.x 
Gardner, R. C., Dams-O'Connor, K., Morrissey, M. R., \& Manley, G. T. (2018). Geriatric Traumatic Brain Injury: Epidemiology, Outcomes, Knowledge Gaps, and Future Directions. J Neurotrauma, 35(7), 889-906. doi:10.1089/neu.2017.5371

Gemechu, J. M., \& Bentivoglio, M. (2012). T Cell Recruitment in the Brain during Normal Aging. Front Cell Neurosci, 6, 38. doi:10.3389/fncel.2012.00038

Gherardin, N. A., Waldeck, K., Caneborg, A. et al (2021). үठ T cells in Merkel cell carcinomas have a proinflammatory profile prognostic of patient survival. Cancer Immunology Research 9, 612-623. https://doi.org/10.1158/2326-6066

Ghoochani, A., Schwarz, M. A., Yakubov, E., Engelhorn, T., Doerfler, A., Buchfelder, M., . . Eyupoglu, I. Y. (2016). MIF-CD74 signaling impedes microglial M1 polarization and facilitates brain tumorigenesis. Oncogene, 35(48), 6246-6261. doi:10.1038/onc.2016.160

Girgis, F., Pace, J., Sweet, J., \& Miller, J. P. (2016). Hippocampal Neurophysiologic Changes after Mild Traumatic Brain Injury and Potential Neuromodulation Treatment Approaches. Front Syst Neurosci, 10, 8. doi:10.3389/fnsys.2016.00008

Gonzalez, H., \& Pacheco, R. (2014). T-cell-mediated regulation of neuroinflammation involved in neurodegenerative diseases. J Neuroinflammation, 11, 201. doi:10.1186/s12974-014-0201-8

Gupta, A., \& Taly, A. B. (2012). Functional outcome following rehabilitation in chronic severe traumatic brain injury patients: A prospective study. Ann Indian Acad Neurol, 15(2), 120-124. doi:10.4103/09722327.94995

Hammond, T. R., Dufort, C., Dissing-Olesen, L., Giera, S., Young, A., Wysoker, A., . . Stevens, B. (2019). Single-Cell RNA Sequencing of Microglia throughout the Mouse Lifespan and in the Injured Brain Reveals Complex Cell-State Changes. Immunity, 50(1), 253-271 e256. doi:10.1016/j.immuni.2018.11.004

Hickman, S., Izzy, S., Sen, P., Morsett, L., \& El Khoury, J. (2018). Microglia in neurodegeneration. Nat Neurosci, 21(10), 1359-1369. doi:10.1038/s41593-018-0242-x

Hopperton, K. E., Mohammad, D., Trepanier, M. O., Giuliano, V., \& Bazinet, R. P. (2018). Markers of microglia in post-mortem brain samples from patients with Alzheimer's disease: a systematic review. Mol Psychiatry, 23(2), 177-198. doi:10.1038/mp.2017.246

Huerta, C., Alvarez, V., Mata, I. F., Coto, E., Ribacoba, R., Martinez, C., . . Pena, J. (2004). Chemokines (RANTES and MCP-1) and chemokine-receptors (CCR2 and CCR5) gene polymorphisms in Alzheimer's and Parkinson's disease. Neurosci Lett, 370(2-3), 151-154. doi:10.1016/j.neulet.2004.08.016

Iba, M., Kim, C., Sallin, M., Kwon, S., Verma, A., Overk, C., . . Masliah, E. (2020). Neuroinflammation is associated with infiltration of T cells in Lewy body disease and alpha-synuclein transgenic models. $\mathrm{J}$ Neuroinflammation, 17(1), 214. doi:10.1186/s12974-020-01888-0 
Islam, M., Davis, B. T. t., Kando, M. J., Mao, Q., Procissi, D., Weiss, C., \& Schwulst, S. J. (2021). Differential neuropathology and functional outcome after equivalent traumatic brain injury in aged versus young adult mice. Exp Neurol, 341, 113714. doi:10.1016/j.expneurol.2021.113714

Izzy, S., Liu, Q., Fang, Z., Lule, S., Wu, L., Chung, J. Y., . . Whalen, M. J. (2019). Time-Dependent Changes in Microglia Transcriptional Networks Following Traumatic Brain Injury. Front Cell Neurosci, 13, 307. doi:10.3389/fncel.2019.00307

Jones, A. R., Overly, C. C., \& Sunkin, S. M. (2009). The Allen Brain Atlas: 5 years and beyond. Nat Rev Neurosci, 10(11), 821-828. doi:10.1038/nrn2722

Joy, M. T., Ben Assayag, E., Shabashov-Stone, D., Liraz-Zaltsman, S., Mazzitelli, J., Arenas, M., .. . Carmichael, S. T. (2019). CCR5 Is a Therapeutic Target for Recovery after Stroke and Traumatic Brain Injury. Cell, 176(5), 1143-1157 e1113. doi:10.1016/j.cell.2019.01.044

Kaech, S. M., Hemby, S., Kersh, E., \& Ahmed, R. (2002). Molecular and functional profiling of memory CD8 T cell differentiation. Cell, 111(6), 837-851. doi:10.1016/s0092-8674(02)01139-x

Kang, S. S., Ebbert, M. T. W., Baker, K. E., Cook, C., Wang, X., Sens, J. P., . . Fryer, J. D. (2018). Microglial translational profiling reveals a convergent APOE pathway from aging, amyloid, and tau. J Exp Med, 215(9), 2235-2245. doi:10.1084/jem.20180653

Karve, I. P., Taylor, J. M., \& Crack, P. J. (2016). The contribution of astrocytes and microglia to traumatic brain injury. Br J Pharmacol, 173(4), 692-702. doi:10.1111/bph.13125

Keane, L., Antignano, I., Riechers, S. P., Zollinger, R., Dumas, A. A., Offermann, N., . . Capasso, M. (2021). mTOR-dependent translation amplifies microglia priming in aging mice. J Clin Invest, 131(20).

doi:10.1172/JCI155208

Keren-Shaul, H., Spinrad, A., Weiner, A., Matcovitch-Natan, O., Dvir-Szternfeld, R., Ulland, T. K., . . . Amit, I. (2017). A Unique Microglia Type Associated with Restricting Development of Alzheimer's Disease. Cell, 169(7), 1276-1290 e1217. doi:10.1016/j.cell.2017.05.018

Lahnemann, D., Koster, J., Szczurek, E., McCarthy, D. J., Hicks, S. C., Robinson, M. D., ... Schonhuth, A. (2020). Eleven grand challenges in single-cell data science. Genome Biol, 21(1), 31. doi:10.1186/s13059020-1926-6

Langlois, J. A., Rutland-Brown, W., \& Wald, M. M. (2006). The epidemiology and impact of traumatic brain injury: a brief overview. J Head Trauma Rehabil, 21(5), 375-378. doi:10.1097/00001199-20060900000001

Lee, M., Lee, E., Han, S. K., Choi, Y. H., Kwon, D. I., Choi, H., . . Lee, Y. J. (2020). Single-cell RNA sequencing identifies shared differentiation paths of mouse thymic innate T cells. Nat Commun, 11(1), 4367.

doi:10.1038/s41467-020-18155-8

Page $17 / 27$ 
Li, T., \& Zhu, J. (2019). Entanglement of CCR5 and Alzheimer's Disease. Front Aging Neurosci, 11, 209. doi:10.3389/fnagi.2019.00209

Loane, D. J., \& Kumar, A. (2016). Microglia in the TBI brain: The good, the bad, and the dysregulated. Exp Neurol, 275 Pt 3, 316-327. doi:10.1016/j.expneurol.2015.08.018

Loane, D. J., Kumar, A., Stoica, B. A., Cabatbat, R., \& Faden, A. I. (2014). Progressive neurodegeneration after experimental brain trauma: association with chronic microglial activation. J Neuropathol Exp Neurol, 73(1), 14-29. doi:10.1097/NEN.0000000000000021

Louveau, A., Plog, B. A., Antila, S., Alitalo, K., Nedergaard, M., \& Kipnis, J. (2017). Understanding the functions and relationships of the glymphatic system and meningeal lymphatics. J Clin Invest, 127(9), 3210-3219. doi:10.1172/JCI90603

Maiden, M. J., Cameron, P. A., Rosenfeld, J. V., Cooper, D. J., McLellan, S., \& Gabbe, B. J. (2020). LongTerm Outcomes after Severe Traumatic Brain Injury in Older Adults. A Registry-based Cohort Study. Am J Respir Crit Care Med, 201(2), 167-177. doi:10.1164/rccm.201903-06730C

Makinde, H. M., Cuda, C. M., Just, T. B., Perlman, H. R., \& Schwulst, S. J. (2017). Nonclassical Monocytes Mediate Secondary Injury, Neurocognitive Outcome, and Neutrophil Infiltration after Traumatic Brain Injury. J Immunol, 199(10), 3583-3591. doi:10.4049/jimmunol.1700896

Masuda, T., Sankowski, R., Staszewski, O., \& Prinz, M. (2020). Microglia Heterogeneity in the Single-Cell Era. Cell Rep, 30(5), 1271-1281. doi:10.1016/j.celrep.2020.01.010

Meng, H., Zhao, H., Cao, X., Hao, J., Zhang, H., Liu, Y., . . Xu, Y. (2019). Double-negative T cells remarkably promote neuroinflammation after ischemic stroke. Proc Natl Acad Sci U S A, 116(12), 5558-5563. doi:10.1073/pnas.1814394116

Moreno-Valladares, M., Silva, T. M., Garces, J. P., Saenz-Antonanzas, A., Moreno-Cugnon, L., Alvarez-Satta, M., \& Matheu, A. (2020). CD8(+) T cells are present at low levels in the white matter with physiological and pathological aging. Aging (Albany NY), 12(19), 18928-18941. doi:10.18632/aging.104043

Moreno-Valladares, M., Silva, T. M., Garces, J. P., Saenz-Antonanzas, A., Moreno-Cugnon, L., Alvarez-Satta, M., \& Matheu, A. (2020). CD8(+) T cells are present at low levels in the white matter with physiological and pathological aging. Aging (Albany NY), 12(19), 18928-18941. doi:10.18632/aging.104043

Mulherkar, S., \& Tolias, K. F. (2020). RhoA-ROCK Signaling as a Therapeutic Target in Traumatic Brain Injury. Cells, 9(1). doi:10.3390/cells9010245

Nasiri, E., Sankowski, R., Dietrich, H., Oikonomidi, A., Huerta, P. T., Popp, J., . . Bacher, M. (2020). Key role of MIF-related neuroinflammation in neurodegeneration and cognitive impairment in Alzheimer's disease. Mol Med, 26(1), 34. doi:10.1186/s10020-020-00163-5 
Ndode-Ekane, X. E., Matthiesen, L., Banuelos-Cabrera, I., Palminha, C. A. P., \& Pitkanen, A. (2018). T-cell infiltration into the perilesional cortex is long-lasting and associates with poor somatomotor recovery after experimental traumatic brain injury. Restor Neurol Neurosci, 36(4), 485-501. doi:10.3233/RNN170811

Nishihara, H., Maeda, M., Tsuda, M., Makino, Y., Sawa, H., Nagashima, K., \& Tanaka, S. (2002). DOCK2 mediates T cell receptor-induced activation of Rac2 and IL-2 transcription. Biochem Biophys Res Commun, 296(3), 716-720. doi:10.1016/s0006-291x(02)00931-2

Ochocka, N., Segit, P., Walentynowicz, K. A., Wojnicki, K., Cyranowski, S., Swatler, J., . . Kaminska, B. (2021). Single-cell RNA sequencing reveals functional heterogeneity of glioma-associated brain macrophages. Nat Commun, 12(1), 1151. doi:10.1038/s41467-021-21407-w

Osier ND, Korpon JR, Dixon CE. Controlled Cortical Impact Model. In: Kobeissy FH, editor. Brain Neurotrauma: Molecular, Neuropsychological, and Rehabilitation Aspects. Boca Raton (FL): CRC Press/Taylor \& Francis; 2015. Chapter 16. Available from: https://www.ncbi.nlm.nih.gov/books/NBK299183/

Panwar, A., Jhun, M., Rentsendorj, A., Mardiros, A., Cordner, R., Birch, K., . . Wheeler, C. J. (2020). Functional recreation of age-related CD8 T cells in young mice identifies drivers of aging- and humanspecific tissue pathology. Mech Ageing Dev, 191, 111351. doi:10.1016/j.mad.2020.111351

Pearson, W. S., Sugerman, D. E., McGuire, L. C., \& Coronado, V. G. (2012). Emergency department visits for traumatic brain injury in older adults in the United States: 2006-08. West J Emerg Med, 13(3), $289-293$. doi:10.5811/westjem.2012.3.11559

Perry, V. H., Nicoll, J. A., \& Holmes, C. (2010). Microglia in neurodegenerative disease. Nat Rev Neurol, 6(4), 193-201. doi:10.1038/nrneurol.2010.17

Pizzolato, G., Kaminski, H., Tosolini, M., Franchini, D. M., Pont, F., Martins, F., . . Fournie, J. J. (2019). Single-cell RNA sequencing unveils the shared and the distinct cytotoxic hallmarks of human TCRVdelta1 and TCRVdelta2 gammadelta T lymphocytes. Proc Natl Acad Sci U S A, 116(24), 11906-11915. doi:10.1073/pnas.1818488116

Ranjan, B., Schmidt, F., Sun, W., Park, J., Honardoost, M. A., Tan, J., . . Prabhakar, S. (2021). scConsensus: combining supervised and unsupervised clustering for cell type identification in single-cell RNA sequencing data. BMC Bioinformatics, 22(1), 186. doi:10.1186/s12859-021-04028-4

Ritzel, R. M., Crapser, J., Patel, A. R., Verma, R., Grenier, J. M., Chauhan, A., . . McCullough, L. D. (2016). Age-Associated Resident Memory CD8 T Cells in the Central Nervous System Are Primed To Potentiate Inflammation after Ischemic Brain Injury. J Immunol, 196(8), 3318-3330. doi:10.4049/jimmunol.1502021 
Roozenbeek, B., Maas, A. I., \& Menon, D. K. (2013). Changing patterns in the epidemiology of traumatic brain injury. Nat Rev Neurol, 9(4), 231-236. doi:10.1038/nrneurol.2013.22

Rusnak, M. (2013). Traumatic brain injury: Giving voice to a silent epidemic. Nat Rev Neurol, 9(4), 186187. doi:10.1038/nrneurol.2013.38

Sala Frigerio, C., Wolfs, L., Fattorelli, N., Thrupp, N., Voytyuk, I., Schmidt, I., . . De Strooper, B. (2019). The Major Risk Factors for Alzheimer's Disease: Age, Sex, and Genes Modulate the Microglia Response to Abeta Plaques. Cell Rep, 27(4), 1293-1306 e1296. doi:10.1016/j.celrep.2019.03.099

Schetters, S. T. T., Gomez-Nicola, D., Garcia-Vallejo, J. J., \& Van Kooyk, Y. (2017). Neuroinflammation: Microglia and T Cells Get Ready to Tango. Front Immunol, 8, 1905. doi:10.3389/fimmu.2017.01905

Schimmel, S. J., Acosta, S., \& Lozano, D. (2017). Neuroinflammation in traumatic brain injury: A chronic response to an acute injury. Brain Circ, 3(3), 135-142. doi:10.4103/bc.bc_18_17

Schwulst, S. J., \& Islam, M. (2019). Murine Model of Controlled Cortical Impact for the Induction of Traumatic Brain Injury. J Vis Exp(150). doi:10.3791/60027

Sebzda, E., Zou, Z., Lee, J. S., Wang, T., \& Kahn, M. L. (2008). Transcription factor KLF2 regulates the migration of naive $T$ cells by restricting chemokine receptor expression patterns. Nat Immunol, 9(3), 292300. doi:10.1038/ni1565

Shi, G. X., Cai, W., \& Andres, D. A. (2013). Rit subfamily small GTPases: regulators in neuronal differentiation and survival. Cell Signal, 25(10), 2060-2068. doi:10.1016/j.cellsig.2013.06.002

Sobel, 2015. R.A. Sobel (2015). Greenfield's Neuropathology (ninth edition), CRC Press.

Streit, W. J., Braak, H., Xue, Q. S., \& Bechmann, I. (2009). Dystrophic (senescent) rather than activated microglial cells are associated with tau pathology and likely precede neurodegeneration in Alzheimer's disease. Acta Neuropathol, 118(4), 475-485. doi:10.1007/s00401-009-0556-6

Szabo, P. A., Levitin, H. M., Miron, M., Snyder, M. E., Senda, T., Yuan, J., .. . Sims, P. A. (2019). Single-cell transcriptomics of human $T$ cells reveals tissue and activation signatures in health and disease. Nat Commun, 10(1), 4706. doi:10.1038/s41467-019-12464-3

Szalay, G., Martinecz, B., Lenart, N., Kornyei, Z., Orsolits, B., Judak, L., . . Denes, A. (2016). Microglia protect against brain injury and their selective elimination dysregulates neuronal network activity after stroke. Nat Commun, 7, 11499. doi:10.1038/ncomms11499

Szulzewsky, F., Arora, S., de Witte, L., Ulas, T., Markovic, D., Schultze, J. L., . . Kettenmann, H. (2016). Human glioblastoma-associated microglia/monocytes express a distinct RNA profile compared to human control and murine samples. Glia, 64(8), 1416-1436. doi:10.1002/glia.23014 
Tatar, C. L., Appikatla, S., Bessert, D. A., Paintlia, A. S., Singh, I., \& Skoff, R. P. (2010). Increased Plp1 gene expression leads to massive microglial cell activation and inflammation throughout the brain. ASN Neuro, 2(4), e00043. doi:10.1042/AN20100016

Todd, B. P., Chimenti, M. S., Luo, Z., Ferguson, P. J., Bassuk, A. G., \& Newell, E. A. (2021). Traumatic brain injury results in unique microglial and astrocyte transcriptomes enriched for type I interferon response. $J$ Neuroinflammation, 18(1), 151. doi:10.1186/s12974-021-02197-w

Todd, K. J., Serrano, A., Lacaille, J. C., \& Robitaille, R. (2006). Glial cells in synaptic plasticity. J Physiol Paris, 99(2-3), 75-83. doi:10.1016/j.jphysparis.2005.12.002

Weyand, C. M., \& Goronzy, J. J. (2016). Aging of the Immune System. Mechanisms and Therapeutic Targets. Ann Am Thorac Soc, 13 Suppl 5, S422-S428. doi:10.1513/AnnalsATS.201602-095AW

Wo, J., Zhang, F., Li, Z., Sun, C., Zhang, W., \& Sun, G. (2020). The Role of Gamma-Delta T Cells in Diseases of the Central Nervous System. Front Immunol, 11, 580304. doi:10.3389/fimmu.2020.580304

Yang, L., Liu, C. C., Zheng, H., Kanekiyo, T., Atagi, Y., Jia, L., . . Bu, G. (2016). LRP1 modulates the microglial immune response via regulation of JNK and NF-kappaB signaling pathways. J Neuroinflammation, 13(1), 304. doi:10.1186/s12974-016-0772-7

Young, K. J., \& Zhang, L. (2002). The nature and mechanisms of DN regulatory T-cell mediated suppression. Hum Immunol, 63(10), 926-934. doi:10.1016/s0198-8859(02)00446-9

\section{Figures}


H\&E

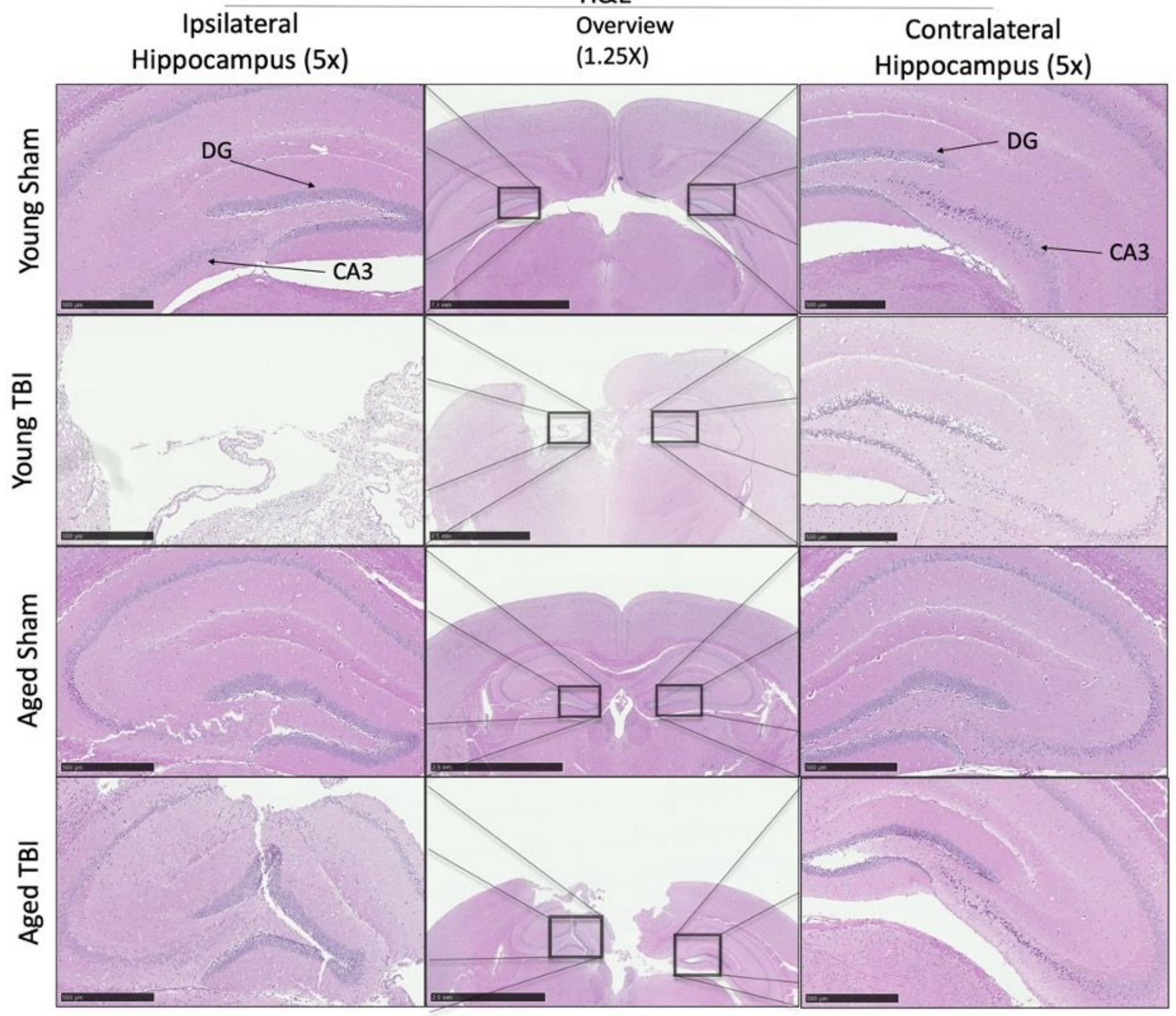

Figure 1

Controlled Cortical Impact induces a severe traumatic brain injury. 10 um Coronal sections stained with hematoxylin and eosin at 30 days post-injury. The center column demonstrates that relative cavity size was similar between young and aged mice. The right and left column represent the ipsilateral and contralateral sides of injury, respectively. $5 x$ magnification reveal more cavitation of the dentate gyrus (DG) and CA3 regions of the hippocampus in young TBI brains as compared to aged TBI brains. There is also greater edema seen in both the ipsilateral and contralateral hemispheres of young TBI brains as compared to aged TBI brains. Sham injury brains demonstrated no difference between young and aged. 
Figure 2

Controlled cortical impact results in greater neuronal loss and astrocytosis in young mice as compared to aged mice after TBI. (A). Neuronal nuclei (NeuN) stained 10 um coronal sections of young and aged mouse brains 30 days post-TBI or sham injury (n-3-5/group). (B). Glial Fibrillary Acidic Protein (GFAP) stained coronal sections of young and aged mouse brains 30 days post-TBI or sham injury (n-3-5/group). (C). \% Degenerated neurons, i.e., \% NeuN-positive cells, in the dentate gyrus and CA3 regions of the hippocampus ( $n=3-5 /$ group; ${ }^{*}<<0.05,{ }^{*} p<0.01$ ). (D). Levels of gliosis (GFAP positivity) within the cortex, hippocampus, and subcortical grey matter in the brains of young and aged mice ( $n=3-5 /$ group; $* *$ $\mathrm{p}<0.01)$. 
A)

IBA1

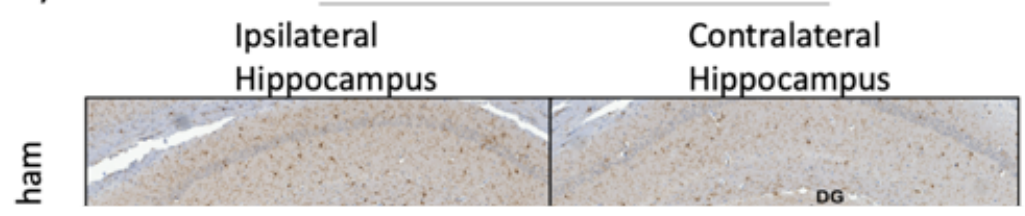

\section{Figure 3}

Controlled cortical impact results in greater IBA1+ staining in aged mouse brains as compared to young mouse brains after TBI. (A). Representative images depicting ionized calcium-binding adaptor molecule 1 (IBA1) stained 10 um coronal sections of young and aged mouse brains 30 days post-TBI or sham injury. Scale bars, $250 \mu \mathrm{m}$. (B). Whole brain levels of IBA1+ microglia. Only aged TBI mouse brains had a 
significant increase in the IBA1 expression within the cortex, hippocampus, and subcortical grey matter (n-3-5/group; $* \star p<0.01, * \star * ~ p<0.001)$.

A

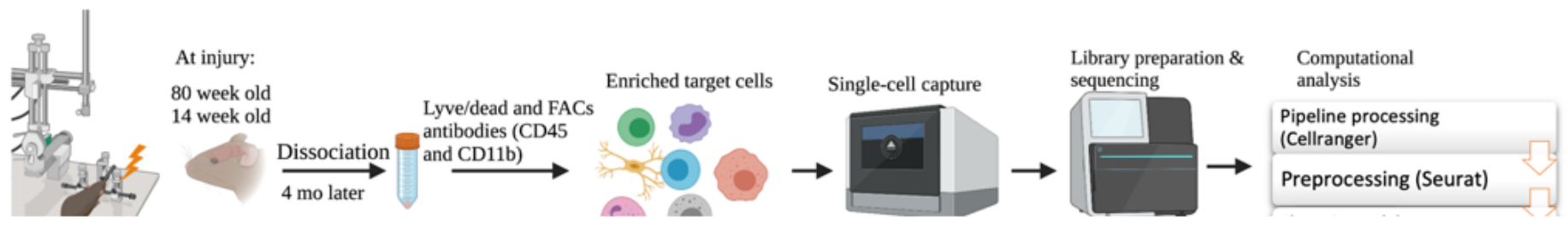

Figure 4

Identification of immune cell populations in sham injured and TBI brains. (A). Scheme of the experimental workflow. Created with BioRender.com. (B) UMAP plot demonstrating clustering obtained for each group (aged sham, aged TBI, young sham, and young TBI), two biological replicates were combined. Cluster annotations: MG microglia, Y.UK young-specific unknown cells, CAM CNS border-associated macrophages, NK natural killer cells, NP neutrophils, MoMФ monocytes/monocytes-derived macrophages. (C) Pie charts demonstrating proportion of the identified cell types across samples. In aged sham mice, the percentages for MG, T, Y.UK, B, CAM\&MФ, NK, NP, MoMФ are 53\%, 28\%, $1.5 \%, 6 \%, 4 \%$, $3.5 \%, 2 \%, 2 \%$. In aged TBI mice, the percentages for MG, T, Y.UK, B, CAM\&MФ, NK, NP, MoMФ are $27.5 \%$, $45.5 \%, 2.5 \%, 11.5 \%, 3.5 \%, 3.5 \%, 4 \%, 2 \%$. In young sham mice, the percentages are $58 \%, 7.5 \%, 28.5 \%, 1.5 \%$, $2 \%, 0.5 \%, 0.5 \%, 2.5 \%$. Lastly, in young TBI mice, the percentages are $71 \%, 4.5 \%, 14 \%, 2 \%, 4 \%, 1 \%, 0.5 \%, 3 \%$.

Figure 5 
Identification of T cell subtypes in sham injured and TBI brains. (A) UMAP plot demonstrating clustering obtained for each $\mathrm{T}$ subtypes, annotated using canonical markers validated by reference datasets curated in ProjecTIL (Andreattae, et al, 2021). T subtype annotations: CD8 ${ }^{+}$EM: CD8 effector memory T cells ( $N=1784), C D 8$ NL: $C D 8$ naïve-like includes $C D 8^{+}$century memory and naïve T cells ( $\left.N=79\right), C D 4$ : CD4 T cells $(N=248), D N T$ : double negative T cells $(N=154)$. (B) Same UMAP plot as in (A) but split to show overall T populations by samples. (C) Stacked bar charts demonstrating proportion of the identified T subtypes across samples. The percentage for each sample in CD8_EM T subset: aged sham $46.4 \%$, aged TBI $51.8 \%$, young sham $0.5 \%$, and young TBI $1.3 \%$. The percentage for each sample in CD4 T subset: aged sham $29.8 \%$, aged TBI $63.3 \%$, young sham $2.4 \%$, and young TBI $4.4 \%$. The percentage for each sample in DNT subset: aged sham $25.3 \%$, aged TBI $68.2 \%$, young sham $3.2 \%$, and young TBI $3.2 \%$. The percentage for each sample in CD8_NL T subset: aged sham $38.0 \%$, aged TBI $39.2 \%$, young sham $15.2 \%$, and young TBI 7.6\%. (D) Volcano plots showing differentially expressed genes of CD8 EM, (E) CD8 NL T cells from aged mice undergoing TBI versus sham. (F) Pathway analysis of differentially expressed genes between TBI and sham CD8 EM T cells in the aged brains with top enriched pathways shown. (G) Pathway analysis of differentially expressed genes between TBI and sham CD8 NL T cells in the aged brains with top enriched pathways shown.

Figure 6

Identification of microglia subclusters in sham injured and TBI brains. (A) UMAP plot demonstrating clustering obtained for microglia population, annotated by their sample origins. Microglia subcluster annotations: Young MG1: young miceenriched microglial subcluster 1 ( $N=952)$, Young MG2: young miceenriched microglial subcluster 2 ( $N=459)$, Aged MG1: aged miceenriched microglial subcluster $1(\mathrm{~N}=584)$, Aged MG2: aged miceenriched microglial subcluster 2 ( $N=1115), M G 1:$ microglial subclusters with no dominant origins ( $\mathrm{N}=2132$ ). (B) Stacked bar charts demonstrating proportion of the annotated microglia subclusters across samples. The percentage for each sample in A.MG2: aged sham $60.4 \%$, aged TBI $21.9 \%$, young sham $6.4 \%$, and young TBI $11.4 \%$. The percentage for each sample in MG1: aged sham $37.1 \%$, aged TBI $14.5 \%$, young sham $32.1 \%$, and young TBI $16.3 \%$. The percentage for each sample in Y.MG1: aged sham 9.0\%, aged TBI 2.4\%, young sham $57.2 \%$, and young TBI $31.3 \%$. The percentage for each sample in Y.MG2: aged sham $2.8 \%$, aged TBI $5.9 \%$, young sham $40.3 \%$, and young TBI $51.0 \%$. The percentage for each sample in A.MG1: aged sham $50.9 \%$, aged TBI $26.9 \%$, young sham $9.4 \%$, and young TBI 12.8\%. (C). Scatter plot depicting expression levels of differentially upregulated genes in Aged TBI and Young TBI. (D). Pathway analysis of upregulated genes expressed by microglia isolated from aged TBI mice with top enriched pathways shown.

\section{Supplementary Files}

This is a list of supplementary files associated with this preprint. Click to download. 
- SupplementalFiguresandMaterialandMethods.docx 